\title{
Virtual Screening of Selected Natural Products as Human Tyrosinase-Related Protein 1 Blocker
}

\author{
Chidi Edbert Duru, ${ }^{\mathrm{a}, *}$, Ijeoma Akunna Duru ${ }^{\mathrm{b}}$, Chiagoziem Wisdom Chidiebere ${ }^{\mathrm{a}}$ \\ ${ }^{a}$ Surface Chemistry and Environmental Technology (SCENT) Research Unit, Department of Chemistry, Imo State University, Owerri, Imo State, Nigeria \\ ${ }^{b}$ Department of Chemistry, Federal University of Technology Owerri, Imo State Nigeria.
}

\begin{abstract}
Many researchers have widely explored the need to replace the harmful compound hydroquinone in skin-lightening creams with more skin-friendly compounds that can give similar results. Some compounds from the plant kingdom have been shown to possess human tyrosinase inhibitory action with no adverse effect on the skin. In this study, the virtual screening of glabridin, kojic acid, arbutin, niacinamide, ascorbic acid, salicin, lactic acid, glutathione, azelaic acid, linoleic acid, glycolic acid, acclaimed to possess this activity as well as the synthetic compound hydroquinone, as human tyrosinase-related protein 1 inhibitor was investigated using computational methods. Site-directed docking was performed at the binding pocket on the enzyme carrying the cocrystallized ligand tropolone. The binding affinity of salicin $(-6.7 \mathrm{kcal} / \mathrm{mol}), \alpha$-arbutin $(-6.3 \mathrm{kcal} / \mathrm{mol})$, glutathione $(-6.2 \mathrm{kcal} / \mathrm{mol})$, ascorbic acid $(-5.7 \mathrm{kcal} / \mathrm{mol})$, and niacinamide $(-5.7 \mathrm{kcal} / \mathrm{mol})$ were higher than that of the cocrystallized ligand tropolone $(-5.5 \mathrm{kcal} / \mathrm{mol})$ and the synthetic skin lightening compound hydroquinone $(-4.8 \mathrm{kcal} / \mathrm{mol})$. $\alpha$-arbutin and glutathione also interacted with similar amino acids units as hydroquinone, suggesting that they followed the exact mechanism of action. These findings strongly corroborate the claim that these natural products could inhibit melanin production and may serve to replace hydroquinone in skin lightening creams.
\end{abstract}

DOI:10.46481/jnsps.2021.253

Keywords: Human tyrosinase-related protein 1; skin lightening; Hydroquinone; Tropolone; Salicin.

Article History :

Received: 16 June 2021

Received in revised form: 2 July 2021

Accepted for publication: 10 July 2021

Published: 29 August 2021

(C)2021 Journal of the Nigerian Society of Physical Sciences. All rights reserved. Communicated by: E. Etim

\section{Introduction}

The pigment melanin majorly impacts the colour of the human skin, hair, and eye. Skin bleaching also known as skin whitening, is applying chemical substances to the skin to make the skin lighter by altering the nature of melanin concentration in the skin [1]. It can also be regarded as the gradual change of the human skin from dark to fair by applying soaps, herbs, chemicals, fade creams, etc., which are strong enough to slow down the function of melanin [2]. Between 25-80\%

${ }^{*}$ Corresponding author tel. no: $+234(0) 8037131739$

Email address: chidiedbertduru@gmail .com (Chidi Edbert Duru ) of Asians and Africans use skin-lightening products to change their skin colour. About $75 \%$ of Nigerian women and between $52-67 \%$ of Senegalese women apply skin bleaching products [3]. A survey conducted in Pretoria, South Africa, reported that $35 \%$ of women in this area apply these products [4]. The three melanogenic enzymes, tyrosinase (TYR), tyrosinase-related proteins (TYRP1), and tyrosinase-related proteins (TYRP2), are required for the biosynthesis of melanin [5]. Difficulties with producing these enzymes in pure form have hampered the understanding of their activity and the effect of mutations that cause albinism and pigmentation disorders [6]. Studies suggest that the TYRP1 enzyme may be responsible for stabilizing 
tyrosinase and determining the shape of melanosomes, which are the structures in melanocytes where melanin is produced. Melanin helps to absorb the UV radiations from the sun and make it fit for people living in the tropical climate of Africa.

Many skin lightening products contain hydroquinone (about $2 \%$ ), which inhibits melanin production in the skin. Studies have shown that this compound has adverse effects such as cancer of melanocytes (melanin cells), contact dermatitis, skin irritation, and exogenous ochronosis mostly rampant in dark-skinned people $[7,8,9]$. Exogenous ochronosis results when the skin is exposed to sun rays over a period of time causing an irregular blue black staining on the skin and nails. It affects the melanin in the skin by inhibiting the polymerization of amino acid tyrosine through oxidation [10]. A resolution to ensure the regulation of the formulation and distribution of beauty products, especially bleaching creams, was recently passed by senators in the Federal Republic of Nigerian [11]. This action was taken to protect Nigerians against the numerous harmful skin whitening cream and soap formulations sold in the market.

Several tyrosinase inhibitors have been tested in cosmetics and pharmaceuticals for preventing excess production of melanin in epidermal layers. Natural products like glabridin, kojic acid, arbutin, niacinamide, ascorbic acid, salicin, lactic acid, glutathione, azelaic acid, linoleic acid, and glycolic acid have been reported to inhibit the action of melanin in the skin [12]. These studies posited that these compounds effectively inhibit melanin formation without any associated cytotoxicity to melanin cells. Reports on applying computational techniques to validate the integrity of these findings are scarce in literature.

In the present study, the inhibitory potentials of these natural products on the human tyrosinase-related protein 1 (TYRP1) were studied in silico as a validation of these claims. Their binding affinity on this enzyme was determined and compared with hydroquinone in the bid to understand their mode of action and identify possible candidates that could replace this synthetic compound in skin lightening products.

\section{Computational Methods}

\subsection{Identification and preparation of ligands}

The 3D structure-data files (SDF) of the selected natural products and hydroquinone were identified and downloaded from the PubChem database. They were minimized in PyRx virtual screening tool, using Universal Force Field at 200 steps followed by their conversion to AutoDock ligands (pdbqt). These files were then used for the docking analysis.

\subsection{Receptor preparation}

The chain A of the human tyrosinase-related protein 1 (PDB ID: $5 \mathrm{M} 8 \mathrm{O}$ ) with resolution $2.50 \AA$ was identified from literature and used as a target for the study. The interfering crystallographic water molecules and cocrystallized ligand were removed, and minimization of the energy of the protein was then done using UCSF Chimera 1.14 [13, 14, 15]. The protein was minimized at 300 steepest descent steps at $0.02 \AA$. The conjugate gradient steps were ten at $0.02 \AA$ and ten update intervals.
Gasteiger charges were also added using Dock Prep to get a good structure conformation [16].

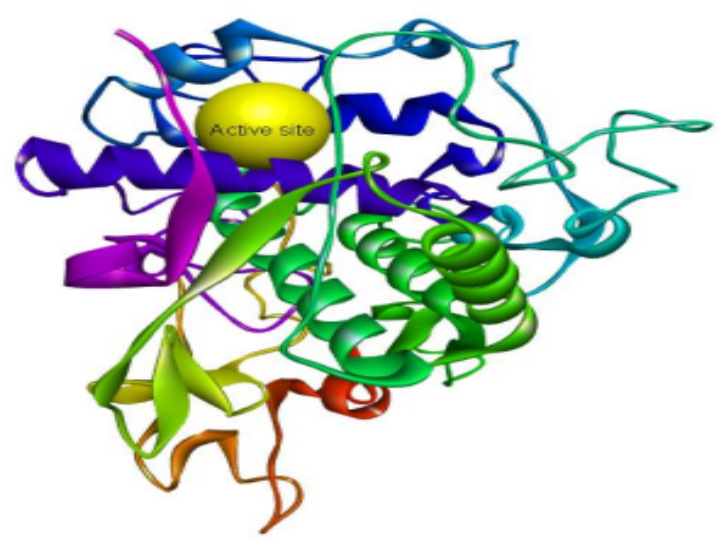

Figure 1. Crystal structure of human tyrosinase-related protein 1 (TRP1) showing binding site

\section{Validation of the docking protocol}

The docking protocol was validated to determine the accuracy and reliability of the docking results. It aimed to accurately reproduce the binding pore and the molecular interactions of the cocrystallized ligand in the protein structure. The native ligand of the $x$-ray protein was downloaded from the PubChem database and minimized in PyRx virtual screening tool. The ligand was then docked into chain A of TYRP1's active site using Auto Dock Vina in PyRx. The docked complex was superimposed with the X-ray resolved crystal TYRP1 downloaded from PDB bearing the cocrystallized ligand to generate the root mean square deviation (RMSD) value in PyMOL. The RMSD value ranging from (0-2) $\AA$ is appropriate for docking and indicates that the protocol could be used to determine the inhibition of the protein by the other small molecules [17].

\subsection{Docking studies}

The multiple ligand docking of the compounds on TYRP1target was done with Autodock Vina in PyRx software version 0.8 $[18,19]$. Site-directed docking was performed at the binding site of the cocrystallized ligand tropolone. The center grid box was set to the dimension center $x:-10.018$, center $y:-1.078$, center $z:-23.140$, and size $x: 19.309$, size $y: 21.549$, size $z: 19.534$. The binding affinity of the compounds was determined in terms of their binding free energy values $(\Delta G)$.

\subsection{Analysis of protein-ligand interactions}

Hydrogen bonding and other hydrophobic interactions between the protein-ligand complex of the compounds were visualized using Biovia Discovery studio 4.5. 
Table 1. Binding affinity values of the natural products on the human TYRP1 enzyme

(liabridin

\section{Results and Discussion}

The docking protocol validation was carried out to assure the deployed docking tools accurately give correct binding interactions between the receptor and the natural products investigated in this study. The docked complex reproduced the original pose of the native ligand (tropolone) with an RMSD value of $1.105 \AA$. The binding affinity values of the natural products on the human TYRP1 are summarized in Table 3.

The binding affinity of salicin $(-6.7 \mathrm{kcal} / \mathrm{mol}), \alpha$-arbutin $(-6.3 \mathrm{kcal} / \mathrm{mol})$, glutathione $(-6.2 \mathrm{kcal} / \mathrm{mol})$, ascorbic acid $(-5.7$ $\mathrm{kcal} / \mathrm{mol})$, and niacinamide $(-5.7 \mathrm{kcal} / \mathrm{mol})$ were higher than
Table 2. Binding interactions of the natural products on the human TYRP1 enzyme

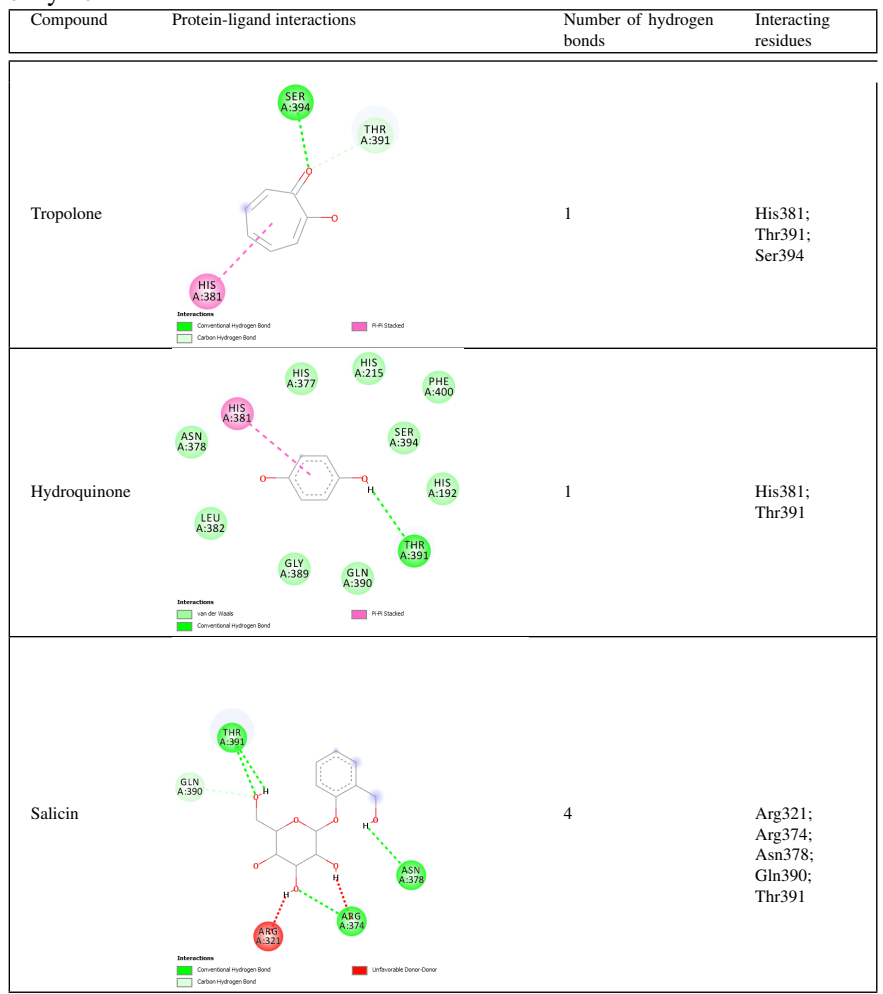

that of the cocrystallized ligand tropolone $(-5.5 \mathrm{kcal} / \mathrm{mol})$ and the synthetic lightening compound hydroquinone $(-4.8 \mathrm{kcal} / \mathrm{mol})$. Salicin is an alcoholic $\beta$-glucoside usually extracted from willow bark. It has been used to treat hyperpigmentation by altering the formation of melanin pigment [20]. $\alpha$-arbutin is commonly extracted from berries and has been reported to reduce skin pigment production. Studies show that $\alpha$-arbutin is safer to apply on the skin than hydroquinone [21]. Glutathione is an antioxidant found in meat and many vegetables like garlic, onion, carrot, potatoes, melon, spinach, etc. Studies have shown that glutathione causes skin whitening by direct inhibition of tyrosinase enzymes [22]. Ascorbic acid, otherwise known as vita$\min C$ is a powerful antioxidant found mostly in citric fruits. It inhibits tyrosine conversion to melanin by tyrosinase and prevents the damaging of the skin by ultraviolet radiation. It improves the appearance of the skin, thereby reducing the rate of aging [23]. Niacinamide, also known as nicotinamide, is a form of vitamin B3 found in meat, fish, nuts, mushrooms, and to a lesser extent in some vegetables. It is a skin-lightening compound that inhibits melanosome transfer from melanocytes to keratinocytes [24, 25].

The interactions of the potent natural product compounds with the human TYRP1 enzyme are shown in Table 2.

The co-crystallized ligand tropolone associated with His 381 , Thr391, and Ser394 forming pi-pi stacked, carbon-hydrogen, and hydrogen bond interactions. Salicin (Thr391), $\alpha$-arbutin (His381; Thr391), glutathione (His381; Thr391; Ser394), ascorbic acid (Ser394), niacinamide (Ser394), and the synthetic com- 
Table 2 Continued

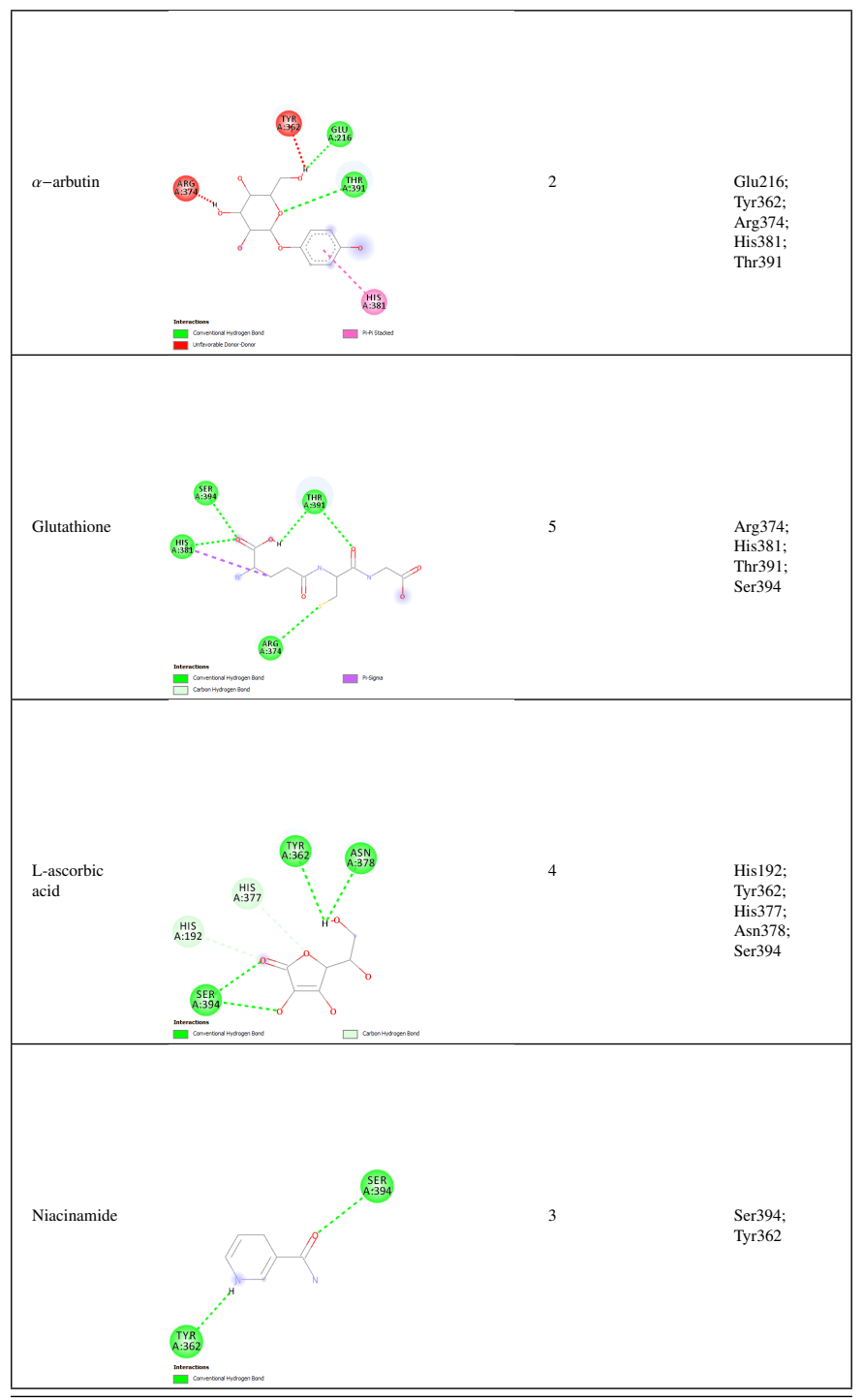

pound hydroquinone (His381; Thr391) also interacted with one or more of these amino acids in the enzyme using similar interactive forces. The hydroxyl $(-\mathrm{OH})$ and the carbonyl $(\mathrm{C}=\mathrm{O})$ functional groups were the predominant moieties that interacted with the amino acid residues in the enzyme. The binding of $\alpha$ arbutin and glutathione on the human TYRP1 enzyme involved His381 and Thr391, which are the two amino acids that bind hydroquinone at this site. This indicated that aside from having a better binding affinity than hydroquinone at this site, they also inhibited melanin production following a similar mechanism as this synthetic compound. The observed number of hydrogen bond interactions between TYRP1 enzyme and salicin (4), $\alpha$ arbutin (2), glutathione (5), ascorbic acid (4), and niacinamide (3) were more than the number in the tropolone (1) and hydroquinone (1) interactions with this enzyme. Hydrogen bond interaction between small molecules and protein sites give the molecules better stability at the binding pockets of the proteins [26]. All the natural product inhibitors studied formed more hy- drogen bonds at the binding site of TYRP1than hydroquinone and indicated that they were more stable at this site than the synthetic inhibitor.

\section{Conclusions}

The in silico validation of the ability of some natural products claimed to possess tyrosinase inhibitory action was performed. Salicin, $\alpha$-arbutin, glutathione, ascorbic acid, and niacinamide gave a higher binding affinity to the human tyrosinase 1 enzyme than the cocrystallized ligand tropolone used as control as well as the synthetic skin lightening compound hydroquinone. $\alpha$-arbutin and glutathione inhibited melanin production following a similar mechanism as hydroquinone and were also more stable at the enzyme site. These findings implicate these natural products as dermatologically active and skin-friendly ingredients that could be used as replacements for hydroquinone in skin bleaching creams.

\section{Acknowledgements}

The authors are grateful to ChemSolvers Research and Computational Laboratory Studio, Owerri, Nigeria, for assisting in the in silico study.

\section{References}

[1] E. Darj, J.J. Infanti, B.M. Ahlberg \& J. Okumu, "The fairer the better? Use of potentially toxic skin bleaching products", Afri Health Sci 15 (2015) 1074.

[2] M. Lartey, F.D. Krampa, M. Abdul-Rahman, N.L. Quarcoo, P. Yamson, P.G. Hagan, Y. Tettey, R. Gyasi \& A.A. Adjei, "Use of skin-lightening products among selected urban communities in Accra, Ghana", Int J Dermatol 56 (2017) 32.

[3] S.B. Adebajo "An epidemiological survey of the use of cosmetic skin lightening cosmetics among traders in Lagos, Nigeria", West Afr J Med 21 (2002) 51.

[4] L.M. Davids, N.P. Van Wyk J. Khumalo \& N.G. Jablonski, "The phenomenon of skin lightening: Is it right to be light?", S Afr J Sci, 112 (2016) 1. http://dx.doi.org/10.17159/sajs.2016/20160056

[5] X. Lai, H.J. Wichers, M. Soler-Lopez \& B.W. Dijkstra, "Structure and function of human tyrosinase and tyrosinase-related proteins", Chemistry 24 (2018) 47.

[6] X. Lai, H.J. Wichers, M. Soler-Lopez \& B.W. Dijkstra, "Structure of human tyrosinase related protein 1 reveals a binuclear zinc active site important for melanogenesis", Angew Chem Int Ed Engl 56 (2017) 9812.

[7] R. Chopra, P.P. Vakharia, R. Sacotte \& J.I. Silverberg, "Efficacy of bleach baths in reducing severity of atopic dermatitis: A systematic review and meta-analysis", Ann Allergy Asthma Immunol 119 (2017) 435.

[8] A. Jose \& R.G. Ray, "Toxic content of certain commercially available fairness creams in Indian market", Cogent Med 5 (2018) 1433104. https://doi.org/10.1080/2331205X.2018.1433104

[9] W. Zhu \& J. Gao, "The use of botanical extracts as topical skin-lightening agents for the improvement of skin pigmentation disorders", J Investig Dermatol Symp Proc. 13 (2008) 20.

[10] T. Pillaiyar, M. Manickam \& V. Namasivayam, "Skin whitening agents: medicinal chemistry perspective of tyrosinase inhibitors", J Enzyme Inhib Med Chem 32 (2017) 403.

[11] Pulse.ng "Senates move to regulate distribution, marketing of bleaching cream", 16 February 2021. https://www.pulse.ng/business/senate-movesto-regulate-bleaching-cream cosmetics/

[12] N. Hanif, A.M. Al-Shami, K.A. Khalid \& H. Ab Hadi, "Plant-based skin lightening agents: A review", J Phytopharmacol 9 (2020) 54. 
[13] E.F. Pettersen, T.D. Goddard, C.C. Huang, G.S. Couch, D.M. Greenblatt, E.C. Meng \& T.E. Ferrin, "UCSF Chimera- a visualization system for exploratory research and analysis", J Comput Chem 25 (2004) 1605.

[14] C.E. Duru, I.A. Duru \& A. Bilar "Computational investigation of sugar fermentation inhibition by bergenin at the pyruvate decarboxylate isoenzyme 1 target of Scharomyces cervisiae", J Med Plants Stud 8 (2020) 21.

[15] C.E. Duru, I.A. Duru, C.B.C. Ikpa, U.E. Enenebeaku, I.C. Obiagwu, M.C. Igbomezie \& M.A. Nnabuchi, "In Silico docking studies of bioactive compounds in Ocimum gratissimum essential oil against Candidapepsin1 Enzyme from Candida albicans", Trop J Nat Prod Res 5 (2021) 364.

[16] BIOVIA DS, "Discovery studio modeling environment", San Diego, Dassault Systemes, Release 20 (2020) 1.

[17] S. Megantara, M.I. Iwo, J. Levita \& S. Ibrahim, "Determination of ligand position in aspartic proteases by correlating tanimoto coefficient and binding affinity with root mean square deviation", J Appl Pharm Sci 6 (2016) 125.

[18] Y.C. Tsao, Y.J. Chang, C.H. Wang \& L. Chen, "Discovery of isoplumbagin as a novel NQO1 substrate and anti-cancer quinine", Int J Mol Sci. 21 (2020) 4378. https://doi.org/10.3390/ijms21124378

[19] I.A. Duru \& C.E. Duru, "Molecular docking of compounds in the essential oil of Ocimium gratissimum leaf against PIM-1 kinase of Escherichia coli”, EJ-CHEM 1 (2020) 1.

[20] L. Hu, L. Zou, Z. Qin, J. Fang, L. Huang \& P. Wang, "A novel label-free bioengineered cell-based biosensor for salicin detection", Sens Actuators
B Chem 238 (2017) 1151.

[21] C. Couteau \& L. Coiffard, "Overview of skin whitening agents: Drugs and cosmetic products", Cosmetics 3 (2016) 27. https://doi.org/10.3390/cosmetics3030027

[22] E.B. Handog, M.S. Datuin \& I.A. Singzon, "An open-label, single-arm trial of the safety and efficacy of a novel preparation of glutathione as a skin-lightening agent in Filipino women”, Int J Dermatol 55 (2016) 153.

[23] R. De Dormael, P. Bastien, P. Sextius, A. Gueniche, D. Ye, C. Tran, V. Chevalier, C.C. Gomes, L. Souverain \& C. Tricaud, "Vitamin C prevents ultraviolet-induced pigmentation in healthy volunteers: Bayesian metaanalysis results from 31 randomized controlled versus vehicle clinical studies", J Clin Aesthet Dermatol 12 (2019) 53.

[24] T. Hakozaki, L. Minwalla, J. Zhuang, M. Chhoa, A. Matsubara, K. Miyamoto, A. Greatens, G.G. Hillebrand, D.L. Bissett \& R.E. Boissy, "The effect of niacinamide on reducing cutaneous pigmentation and suppression of melanosome transfer", Br J Dermatol 147 (2002) 20.

[25] N. Iftekhar \& V.P. Zhitny, "Overview of skin bleaching history and origins", Dermatology 237 (2021) 306.

[26] C.E. Duru, I.A. Duru \& A.E. Adegboyega "In Silico identification of compounds from Nigella sativa seed oil as potential inhibitors of SARS-CoV-2 targets", Bull Natl Res Cent 45 (2021) 57. https://doi.org/10.1186/s42269-021-00517-x 